\title{
ANGULAR SELECTION OF PRODUCTS BY USE OF SATURATED-ABSORPTION TECHNIQUES IN THE Cs(7P) $+\mathrm{H}_{2} \rightarrow \mathrm{CsH}+\mathrm{H}$ REACTION
}

\author{
J.-M. L'HERMITE, G. RAHMAT and R. VETTER \\ Laboratoire Aimé Cotton, C.N.R.S. II Bat. 505, 91405 Orsay-Cedex, \\ France
}

(Received 20 February, 1990; in final form 1 March, 1990)

\begin{abstract}
The $\mathrm{Cs}(7 \mathrm{P})+\mathrm{H}_{2} \rightarrow \mathrm{CsH}+\mathrm{H}$ reaction is studied in a crossed-beam experiment with laser-induced fluorescence detection of $\mathrm{CsH}$ products. The usual flux $\left(\approx 50 \mathrm{~mW} / \mathrm{mm}^{2}\right)$ which is delivered by the C.W. tunable dye laser used in the experiment is enough to saturate the absorption by $\mathrm{CsH}$ products. Then, by crossing twice the laser beam through the collision volume (counterpropagating beams), one realizes the conditions of saturated-absorption experiments: when the laser frequency is tuned to a resonance frequency of $\mathrm{CsH}$ products, a defect to absorption occurs for these products which scatter in the collision plane and a "saturation dip" appears at the center of the corresponding fluorescence profile. Application of this technique to crossed-beam experiments can lead to the selection of product molecules which scatter in any definite plane. A different geometry of the laser beams (bent beams) is proposed to select molecules which scatter in any definite direction: it could be applied to detect an asymmetry in the scattering of the products with respect to the collision axis, when a particular preparation of the reagents is realized.
\end{abstract}

KEY WORDS: Reactive collisions, crossed-beam experiments, laser-induced fluorescence, Doppler technique, saturated-absorption, angular selection of products.

\section{INTRODUCTION}

It is now well established that the use of the laser-induced fluorescence (L.I.F.) technique to detect reaction products in crossed-beam experiments offers a number of advantages: ${ }^{1}$ experimental simplicity, high sensitivity and high resolution essentially. First it allows for measurement of reactive cross sections for products in definite rovibrational levels; second it allows, when C.W. lasers are used, for the spectral analysis of fluorescence profiles, hence high resolution informations on the velocity and the angular scattering probability of reaction products, directly in the center of mass (Doppler technique).$^{2-6}$ In cases where the laser intensity is large enough to saturate the absorption by product molecules, the use of classical saturated-absorption techniques increases the spectral resolution, allowing for example the identification of hyperfine components in fluorescence signals. ${ }^{7,8}$ Here, we present another application of saturated-absorption techniques to crossed-beam experiments: the selection of reaction products which scatter in definite planes or in definite directions.

In classical saturated-absorption experiments conducted in gas cells at low pres- 
sure,$^{9,10}$ the aim is to eliminate the Doppler broadening which generally limits the spectral resolution. In these experiments, a tunable laser beam at frequency $v$ is used to saturate the absorption of molecules (or atoms) whose velocity component along the propagation direction of the beam, $V_{x}$, follows the Doppler relation:

$$
v-v_{0}=v_{0} V_{x} / c
$$

where $v_{0}$ is the molecular resonance frequency and $c$ the velocity of light. A counterpropagating probe beam at the same frequency is absorbed by the molecules whose velocity component is $-V_{x}$. As the laser frequency is scanned, one observes at $v=v_{0}$ a weaker absorption of the probe beam since the absorption by molecules with $V_{x}=0$ is already saturated. The dip which appears has a Lorentzian shape, its width is the natural width broadened by saturation. ${ }^{9-10}$ Usual experimental techniques (modulation of the saturation beam and lock-in detection on the probe) allow for separation and recording of the dip itself: saturation spectroscopy is thus a means to perform high resolution Doppler-free measurements. Collisional studies can also be performed. ${ }^{11,12}$

\section{COUNTERPROPAGATING BEAM ARRANGEMENT IN $\mathrm{Cs}^{*}+\mathrm{H}_{2} \rightarrow \mathrm{CsH}+\mathrm{H}$ EXPERIMENTS}

In the high resolution crossed-beam experiment devoted to the study of the $\mathrm{Cs}(7 \mathrm{P})+\mathrm{H}_{2} \rightarrow \mathrm{CsH} \mathrm{X}{ }^{1} \Sigma^{+}\left(v^{\prime \prime}=0, J^{\prime \prime}\right)+\mathrm{H}$ photochemical reaction, ${ }^{6,13-16}$ a first C.W. laser beam is used to excite Cs atoms to a given $\left(7 P_{1 / 2}, F^{\prime}\right)$ hyperfine level and a second, tunable, C.W., collinear laser beam is used to detect $\mathrm{CsH}$ products, through the L.I.F. technique. In the experimental results presented here, the two laser beams are adjusted perpendicularly to the collision plane. A precise and slow scanning (1MHz/s typically) of the second laser frequency over the absorption profile of a given $\mathrm{X}^{1} \Sigma^{+}\left(v^{\prime \prime}=0, J^{\prime \prime}\right) \rightarrow \mathrm{A}^{1} \Sigma^{+}\left(v^{\prime}=5, J^{\prime}=J^{\prime \prime}+1\right)$ transition of CsH molecules yields a fluorescence profile $\mathrm{A}(v)$, shown on Figure 1a for $J^{\prime \prime}=6$. Its area represents the reactive cross section relative to $J^{\prime \prime}$ and its shape gives information on the velocity and the angular scattering probability of reaction products. ${ }^{6}$

In our experiment, we observed a saturation of the $\mathrm{CsH}$ absorption, the fluorescence signal being proportional to the square root of the laser flux, usually between 40 and $100 \mathrm{~mW} / \mathrm{mm}^{2}$ : the excitation of $\mathrm{CsH}$ products is performed in a coherent saturation regime $\mathrm{e}^{5}$ and the $\mathrm{CsH}$ absorption is proportional to the Rabi frequency $f_{1}$, thus to the laser field. By sending the analysis laser beam back to the collision volume (by a simple reflection on a mirror) we observed, as expected, a "saturation dip" at the central frequency $v_{0}$ of the fluorescence profile $B(v)$ : it is shown on Figure 1b.

In this reactive system, the reagent energy is well determined: thus, all CsH molecules which are detected on a definite rotational level have the same kinetic energy, thus the same modules $w$ of the velocity in the center of mass. ${ }^{6,13-16}$ Figure 2 explains the formation of the dip. For $v \neq v_{0}$, the molecules which absorb the radiation are those which scatter with a velocity component $w_{x}$ along the "primary" 

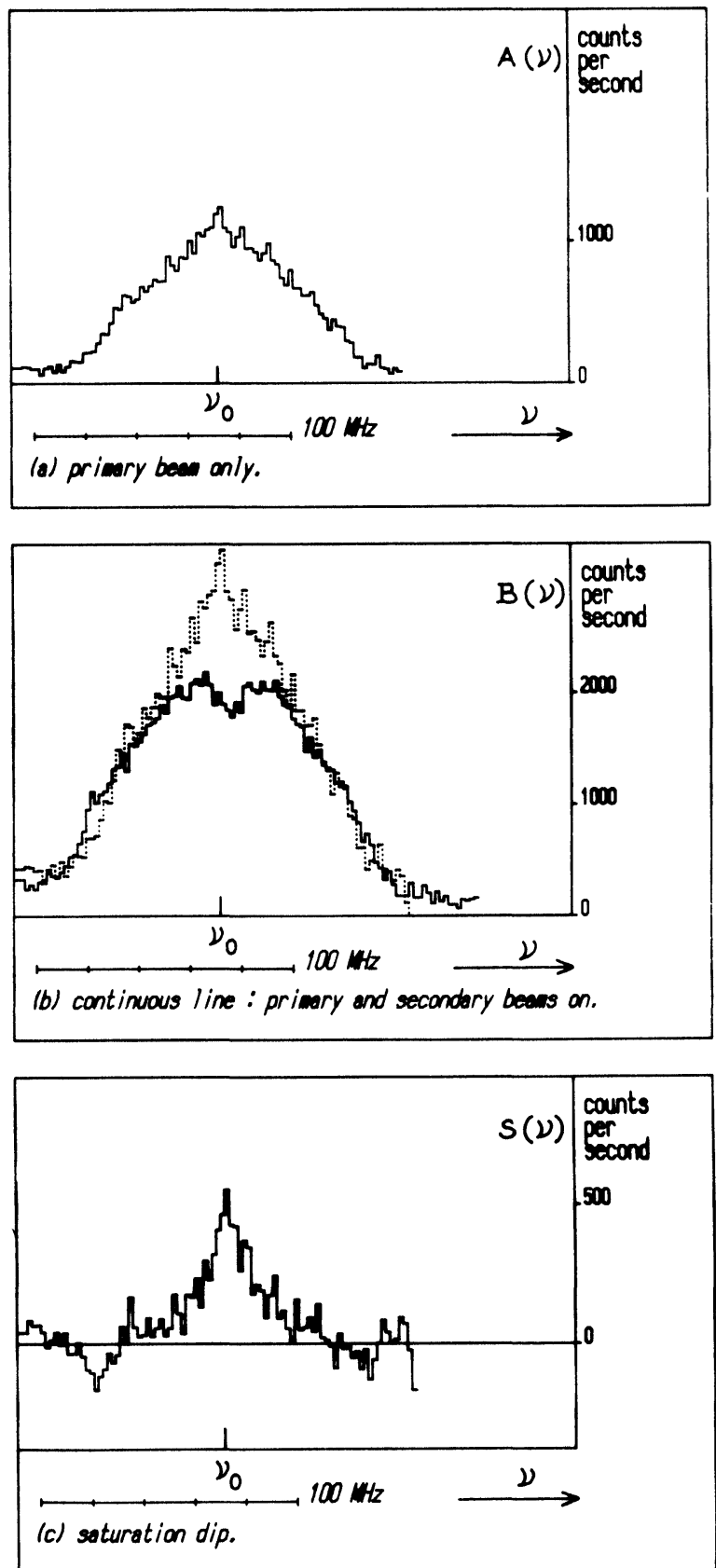

Figure 1 Fluorescence profiles of the $\mathrm{X}^{1} \Sigma^{+}\left(v^{\prime \prime}=0, J^{\prime \prime}=6\right) \rightarrow \mathrm{A}^{1} \Sigma^{+}\left(v^{\prime}=5, J^{\prime}=7\right)$ line of CsH. Each point of recorded profiles corresponds to integration of the fluorescence signal for one second. (a) $A(v)$ : primary beam only. (b) $B(v)$ (continuous line): "primary" and "secondary" beams on. Dots correspond to $2 A(v)$. (c) Saturation dip $S(v)$, obtained by subtracting the two profiles of Figure 1b (i.e. $S(v)=$ $2 A(v)-B(v))$. 


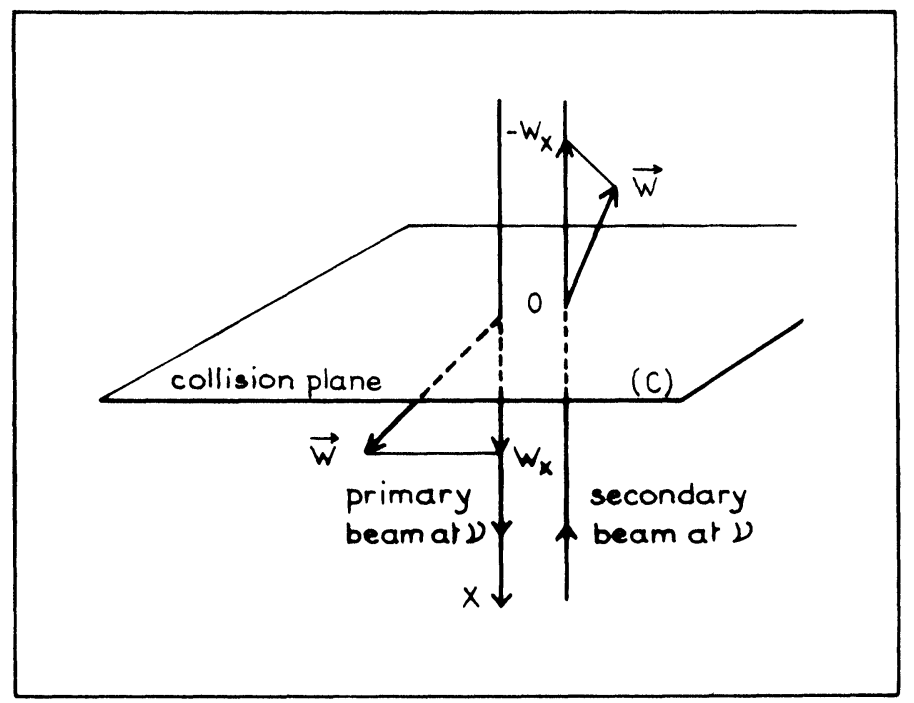

Figure 2 Schematic diagram of the beam arrangement; the "secondary" laser beam is collinear with the "primary" one (counterpropagating waves).

laser beam, according to expression (1); the molecules which absorb the radiation of the "secondary" laser beam have a velocity component $-w_{x}$. As products scatter symmetrically with respect to the collision plane (C), the total signal $B(v)$ is roughly twice the signal $A(v)$ relative to one single beam. For $v=v_{0}$, the molecules which absorb the two counterpropagating beams correspond to $w_{x}=0$, they scatter in the collision plane: due to saturation, the magnitude of the signal is $\sqrt{2 A}\left(v_{0}\right)$ only and a dip occurs in the fluorescence profile at the resonance frequency.

Figure 1c shows the saturation dip $S(v)=2 A(v)-B(v)$, extracted from the two curves of Figure $1 \mathrm{~b}$. It can be approximated by a Lorentzian, whose width $\delta v \approx 20-25 \mathrm{MHz}$ (F.W.H.M.) is mainly due to the natural width $(\Gamma \approx 14 \mathrm{MHz}$ ) of the molecular transition broadened by saturation: in our coherent saturation regime. the saturation width is equal to $\delta v_{R}=f_{1} \sqrt{\Gamma \tau}=22 \mathrm{MHz}$, where $f_{1}=4.8 \mathrm{MHz}^{17}$ and $\tau=1.510^{-6} \mathrm{~s}$ is the mean transit time of $\mathrm{CsH}$ products in the laser field. The jitter of the laser and the Doppler broadening associated with the angular divergence of the laser beams being of the order of $5 \mathrm{MHz}$ each, it appears that $\delta v$ and $\delta v_{R}$ are in good agreement.

The shape and width of the saturation dips that we recorded refer to molecules which scatter around the collision plane, with a non-negligible velocity dispersion along the laser axis. In the general case where two counterpropagating beams of same energy and same frequency are used, the saturation dip has the same shape and the same width as the interaction between molecules and one single laser beam..$^{9,10}$ In our case (Figure 3), absorption occurs for two symmetrical bands of molecules whose velocity dispersion is a Lorentzian of width $\delta w_{x}$; saturation occurs when the two bands overlap, in a central zone whose velocity dispersion is also a Lorentzian of 

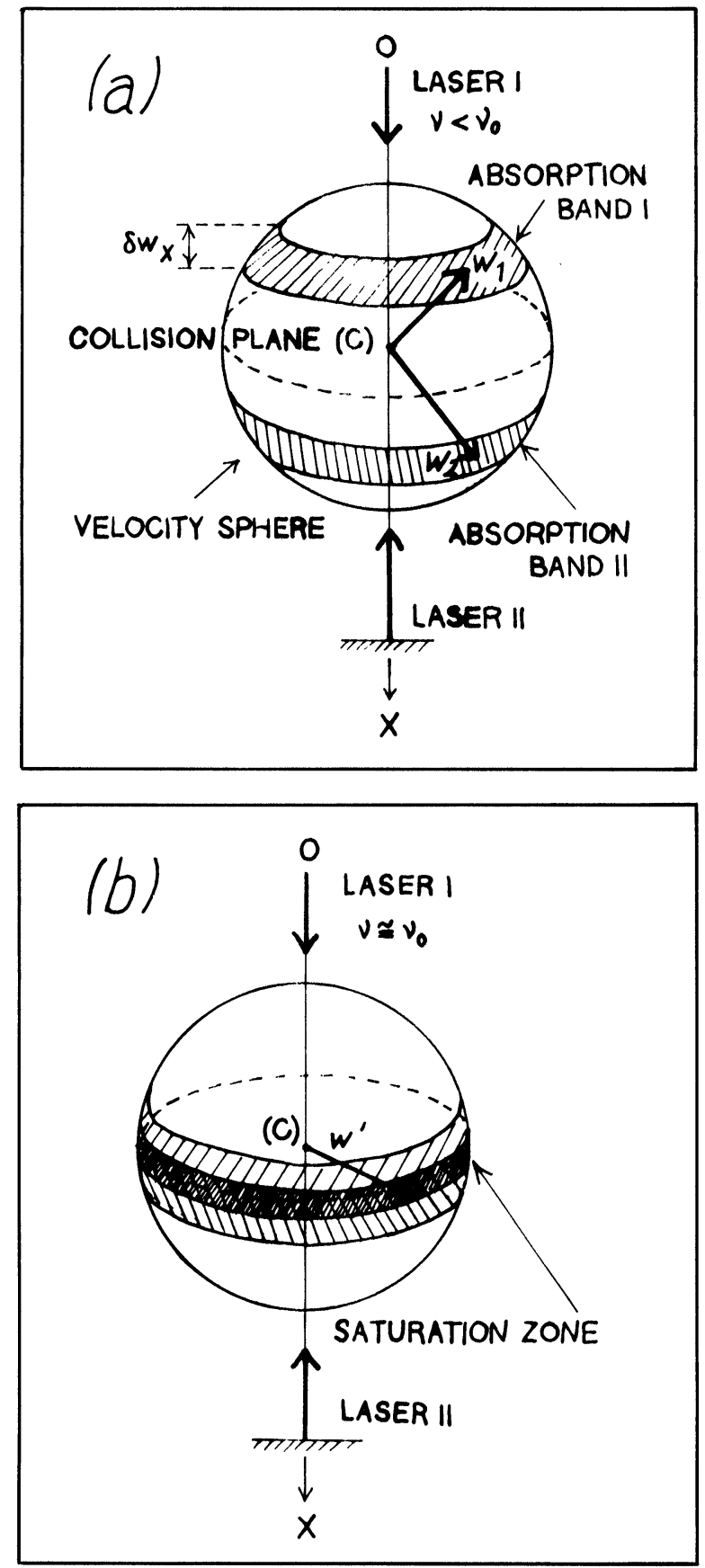

Figure 3 Velocity sphere in the center of mass showing: (a) the two absorption bands (width $\delta w_{\mathrm{x}}$ ), for $v<v_{0}$; (b) the saturation zone, for $v \approx v_{0}$, when the two bands overlap around the collision plane. $\vec{w}^{\prime}$ is the velocity of molecules which absorb beam I and beam II simultaneously. 
width $\delta w_{x}$ which is connected to the spectral width of the saturation dip by the following relation:

$$
\delta w_{x} / \mathrm{w}=(c / w)\left(\delta v / v_{0}\right)=2(\delta v / \Delta v)
$$

where:

$$
\Delta v=2 w v_{0} / c
$$

is the full Doppler width. $\delta w_{x} /$ w represents the angular width (in radian) of the class of molecules (around the collision plane) which absorb the two beams simultaneously.

For the $\mathrm{Cs}(7 \mathrm{P})+\mathrm{H}_{2} \rightarrow \mathrm{CsH}+\mathrm{H}$ photochemical reaction which is operated at threshold, ${ }^{6,13-16}$ the kinetic energy available to $\mathrm{CsH}$ products is very small and so is their velocity in the center of mass: for $J^{\prime \prime}=6 ; w=28 \mathrm{~m} / \mathrm{s}$, the corresponding full Doppler width is $\Delta v=100 \mathrm{MHz}\left(3.310^{-3} \mathrm{~cm}^{-1}\right)$. With $\delta v=25 \mathrm{MHz}$, the angular width $\delta w_{x} / w$ corresponds to an angle of $\pm 15^{\circ}$, typically. Saturation dips were observed for other $J^{\prime \prime}$ values and led roughly to the same parameters.

Since the use of modulation techniques allows for recording of the dip itself, this saturation technique offers a means to select molecules which scatter in the collision plane. Any other scattering plane could be selected by a proper orientation of the two counterpropagating laser beams.

\section{BENT BEAM ARRANGEMENTS}

In the particular case where the velocity in the center of mass, $w$, is unique for all detected products, one has the possibility, also, to select some particular directions of scattering, by use of a bent beam arrangement. Consider the case of Figure 4a: the "primary" beam (beam I along $\mathrm{OX}_{1}$ ) and the "secondary" beam (beam II along $\mathrm{OX}_{2}$ ) cross at the collision volume with an angle $\gamma$ : they are in the plane (L). $\vec{w}$ fixed by its angle $\beta$ with respect to $\mathrm{OZ}$ and by the angle $\alpha$ of its projection over (L) with respect to $\mathrm{OX}_{1}$. We choose the particular case where the velocity of the center of mass (along OZ) is perpendicular to (L). Figure $4 \mathrm{~b}$ represents the velocity sphere in the center of mass: products which absorb the radiation of beam I at the frequency $v$ scatter with $\vec{w}$ pointing on circle I and products which absorb the radiation of beam II (at the same frequency) point on circle II. Saturation occurs for molecules whose velocity vectors $\vec{w}^{\prime}$ and $\vec{w}^{\prime \prime}$ point at the intersection of the two circles, i.e., those for which the projections of $\vec{w}$ over the two laser axis, $w_{x 1}$ and $w_{x 2}$, verify the Doppler relation:

$$
w_{x 1}=w_{x 2}=c\left(v-v_{0}\right) / v_{0}
$$

As $w_{x 1}=w \sin \beta \cos \alpha$ and $w_{x 2}=w \sin \beta \cos (\alpha-\gamma)$, the relation (4) is verified for one particular value of $\alpha$ equal to $\gamma / 2$. Two distinct values of $\beta, \beta^{\prime}$ and $\beta^{\prime \prime}=\pi-\beta^{\prime}$ are solutions of relation (4), with:

$$
\beta^{\prime}(v)=\arcsin \left[\frac{2\left(v-v_{0}\right)}{\Delta v \cos (\gamma / 2)}\right]
$$



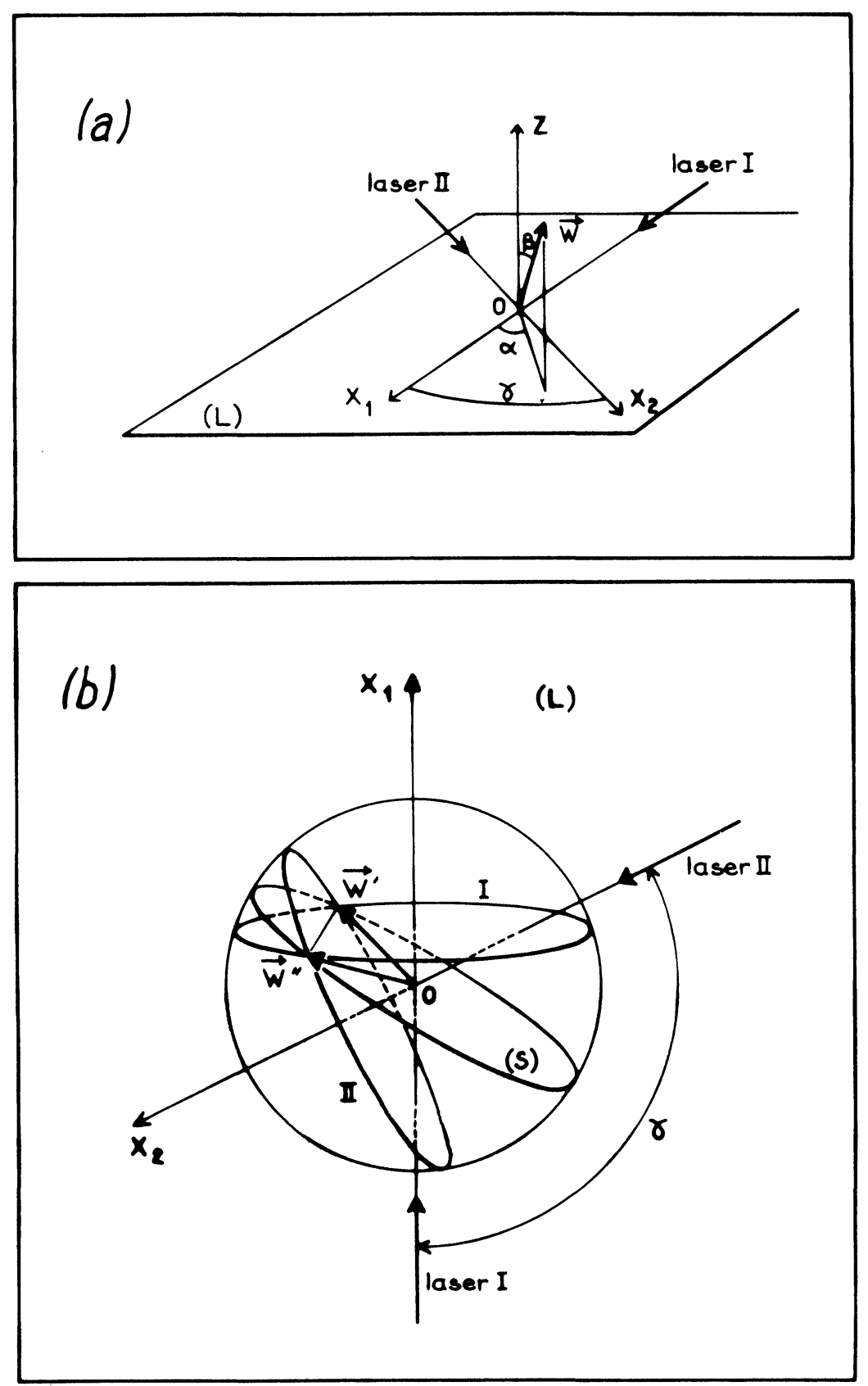

Figure 4 Bent beam arrangement. (a) the two laser beams make an angle $\gamma$ and the velocity of the center of mass is along $\mathrm{OZ}, \vec{w}$ is the velocity of products in the centre of mass. (b) velocity sphere in the center of mass; molecules which absorb simultaneously the two beams point their velocity vectors $\vec{w}^{\prime}$ and $\vec{w}^{\prime \prime}$ on the circle $(S)$, bisector of the planes $\left(\mathrm{ZOX}_{1}\right)$ and $\left(\mathrm{ZOX}_{2}\right)$. 

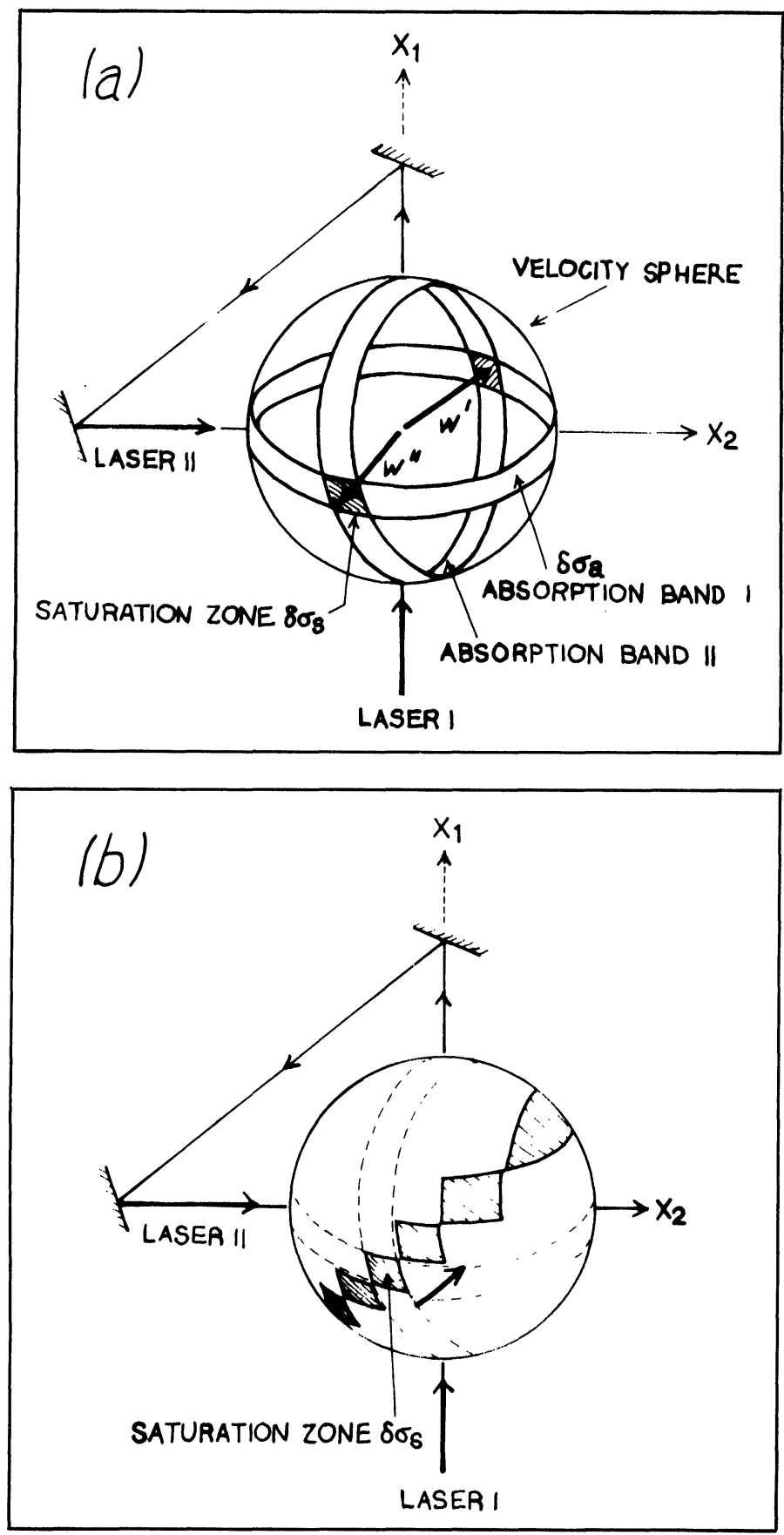

Figure 5 Bent beam arrangement, with $\gamma=90^{\circ}$. (a) the two absorption bands (width $\delta w_{x}$, area and $\delta \sigma_{a}$ ) intersect in two saturation zones (area $\delta \sigma_{s}$ ). (b) saturation zones more on the velocity sphere when one increases the laser frequency step by step, by the quantity $\delta v$. 
Consequently, the choice of $\gamma$ defines the particular circle $(S)$ on the velocity sphere where saturation occurs $((S)$ is bisector of circles I and II). The angle $\beta$ can be chosen between 0 and $180^{\circ}$ by adjusting the laser frequency according to expression (5), between the limits:

$$
v_{0}-(\Delta v / 2) \cos (\gamma / 2) \leq v \leq v_{0}+(\Delta v / 2) \cos (\gamma / 2) .
$$

Outside these limits, circle I and circle II do not intersect and no saturation occurs. At the limits of $v$ indicated above, only one velocity is selected since $\beta^{\prime}=\beta^{\prime \prime}=\pi / 2$ $(\vec{w}$ is in the plane (L)).

In practice, due to the width of the interaction between molecules and radiation, absorption occurs for two bands of same width $\delta w_{x}=2 w(\delta v / \Delta v)$ and of same area $\delta \sigma_{a}=4 \pi w^{2}(\delta v / \Delta v)$ : actually, to simplify calculations of the final angular resolution, we will use in the following a constant interaction over the frequency range $\delta v$ (in that case, the saturation dip $S(v)$ would be triangular instead of Lorentzian). Saturation occurs in two zones of the velocity sphere where the two bands intersect (Figure 5a, in the particular case where the two laser beams are perpendicular, $\gamma=90^{\circ}$ ). In the most general case, these two zones are diamondshaped, with two diagonal lengths $w \delta \alpha$ and $w \delta \beta$; their area is $\delta \sigma_{\mathrm{s}} \approx w^{2} \delta \alpha \delta \beta / 2$. The relative positions of these zones on the velocity sphere are indicated on Figure $5 b$ for various values of the laser frequency with respect to the resonance frequency.

In the case where $\delta v$ is small compared with $\Delta v$ :

$$
\begin{aligned}
& \delta \alpha=2(\delta v / \Delta v) / \sin (\gamma / 2) \\
& \delta \beta=2(\delta v / \Delta v) /(\cos (\gamma / 2) \cos \beta) \\
& \delta \sigma_{s}=4 w^{2}(\delta v / \Delta v)^{2} /(\sin \gamma \cos \beta)
\end{aligned}
$$

When $\beta$ varies from $0^{\circ}$ to $90^{\circ}$, the area $\delta \sigma_{s}$ selected on the velocity sphere increases, hence the angular resolution decreases.

In the case where $\delta v$ is not small compared with $\Delta v$, it is necessary to perform an integration over the velocity sphere to obtain $\delta \sigma_{s}$; in the case of $\gamma=90^{\circ}$, it reduces to:

$$
\delta \sigma_{s}=4 \mathrm{w}^{2}(\delta v / \Delta v)^{2} \int_{x-\delta x}^{x+\delta x} \mathrm{~d} x \int_{x-\delta x}^{x+\delta x} \mathrm{~d} y\left(1-x^{2}-y^{2}\right)^{-1 / 2}
$$

where $x=y=w_{x 1} / w=2\left(v-v_{0}\right) / \Delta v$ and $\delta x=(\delta v / \Delta v)$.

The quantities $\delta \alpha, \delta \beta$ and $\delta \sigma_{a}$ are quoted on Figures $6 \mathrm{a}$ and $6 \mathrm{~b}$, versus the laser frequency and the corresponding angle $\beta$, for three values of the ratio $\delta v / \Delta v: 0.05$, 0.15 and 0.25 (this last value corresponds to our experimental situation, for $J^{\prime \prime}=6$ ). As expected, $\delta \alpha$ remains constant with $\beta, \delta \beta$ increases rapidly with $\beta$ : consequently, the final angular resolution increases with $\beta$, since $\delta \sigma_{s}$ follows approximately the variation of $\delta \beta$. In the case of an isotropic scattering of products, the magnitude of the saturation dip with respect to the absorption signal due to a single laser beam is $Q=2(1-1 / \sqrt{2})\left(\delta \sigma_{s} / \delta \sigma_{a}\right)$. As $\left(\delta \sigma_{s} / \delta \sigma_{a}\right)$ is proportional to $\delta v / \Delta v$, the saturation signal is small when the angular resolution is high. 

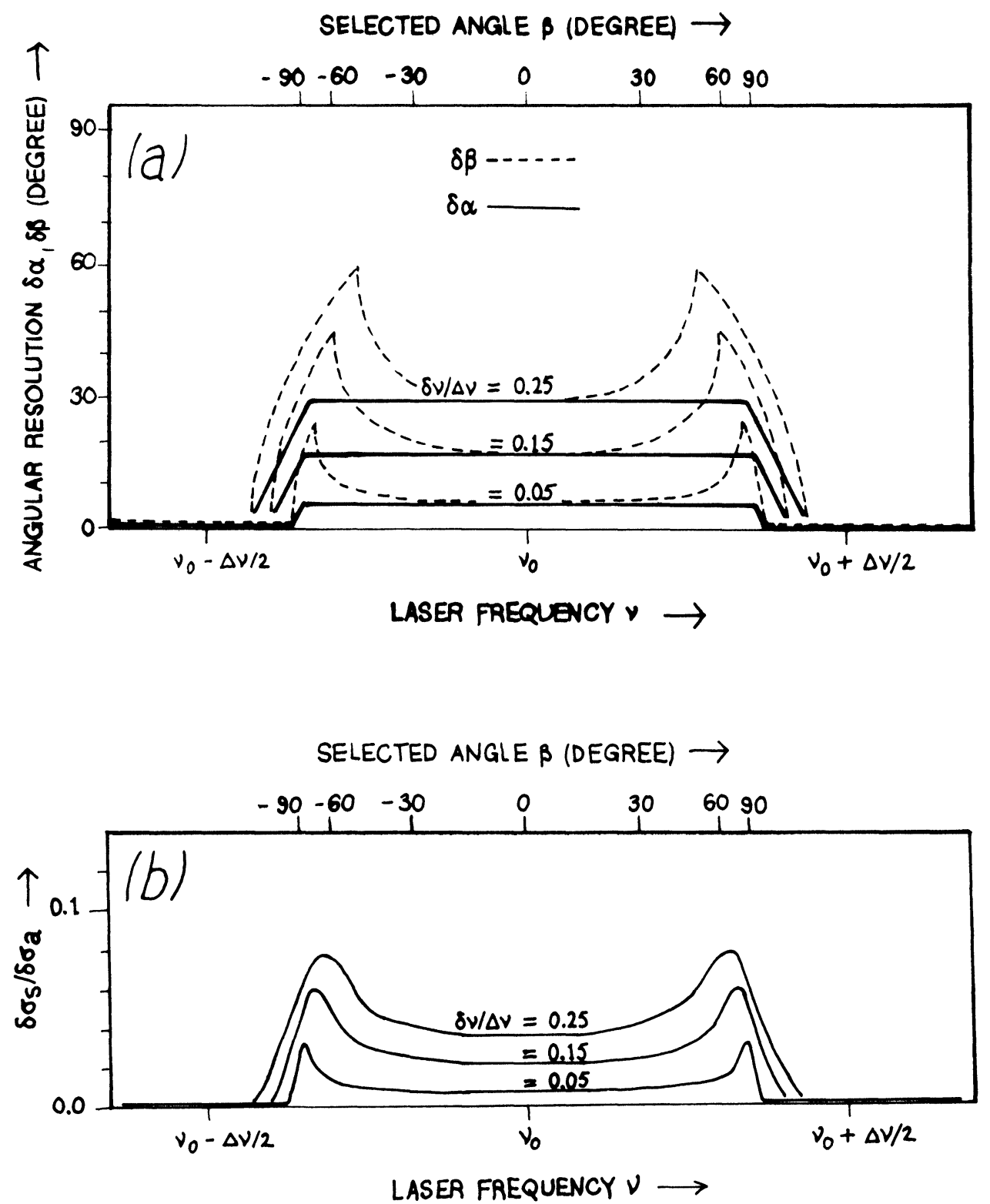

Figure 6 Variations of $\delta \alpha, \delta \beta$ (Fig. $6 a$ ) and $\delta \sigma_{s} / \delta \sigma_{a}$ (Fig. $6 b$ ) versus the laser frequency and the selected angle $\beta$ for three values of the spectral width $\delta v$. The angular resolution is best at small $\beta$ values. 


\section{DISCUSSION}

Saturated-absorption using this bent arrangement could be of interest in crossedbeam experiments, to determine reactive scattering differential cross sections from the shape of fluorescence profiles. In the case where it is not possible to adjust the analysis laser beam along the collision axis (the most general case), at least two recordings of fluorescence profiles with two different orientations of the laser beam are necessary to obtain the angular scattering probability without ambiguity (even if the product velocity is unique) ${ }^{2-6}$ In saturated-absorption experiments using this bent arrangement, one obtains directly, at a given frequency, the contribution of only two scattering directions which reduce to one for $\beta=90^{\circ}$. Another application of this saturation technique is offered for experiments where it is essential to select one particular direction of scattering: for example, for experiments on alignment/ orientation of reagents where it was shown that the scattering may not be symmetrical with respect to the collision axis. ${ }^{18}$

These experiments can be realized only if the fluorescence signal is large enough because the saturation signal itself corresponds to a small fraction of the scattering products. The saturation signal with a counterpropagating beam arrangement is evidently larger than the saturation signal with a bent beam arrangement since the former corresponds to all products which scatter in a given plane and the latter to products which scatter in two directions only. The cross section of the $\mathrm{Cs}(7 \mathrm{P})+\mathrm{H}_{2} \rightarrow \mathrm{CsH}+\mathrm{H}$ reaction is small, $10^{-16} \mathrm{~cm}^{2}$ approximatively: $:^{13-16}$ under our experimental conditions, the fluorescence signal corresponds at best to a few thousands counts/s only and the saturation signal is just a little larger than the statistical noise (see Figure 1). For this reason, we did not try to perform polarization experiments ${ }^{19}$ with this bent beam arrangement. Moreover, due to the small velocity of $\mathrm{CsH}$ products in the center of mass, Doppler profiles are very narrow, only three or four times broader than the "apparatus function" of the experiment whose width is of the order of $\delta v$ typically: this limits the final angular resolution and restricts the interest of the bent beam arrangement. Better conditions could be found in inelastic scattering studies where cross sections and product velocities are much larger.

\section{References}

1. R. B. Bernstein, Chemical Reactivity via Molecular Beam and Laser Techniques, Oxford University Press, Oxford (1982).

2. J. L.Kinsey, J .Chem. Phys., 66, 2560 (1977).

3. J. A. Serri, J. L. Kinsey and D. E. Pritchard, J. Chem. Phys., 75, 663 (1981).

4. E. J. Murphy, J. H. Brophy, G. S. Arnold, W. L. Dimpfl and J. L. Kinsey, J. Chem. Phys., 70, 5910 (1979).

5. N. Billy, B. Girard, G. Gouédard and J. Vigué, Mol. Phys., 61, 65 (1987) and Laser Chem., 93, 434 (1990).

6. J.-M. L'Hermite, G. Rahmat and R. Vetter, J. Chem. Phys., 93. 434 (1990).

7. G. Meijer, W. Ubachs, J. J. ter Meulen and A. Dymanus, Abstracts of the XIth International Symposium on Molecular Beams, Edinburgh, p. 483 (1987).

8. B. Girard, N. Billy, G. Gouédard and J. Vigué, Abstracts of the VIIth European Conference on the Dynamics of Molecular Collisions, Assisi, p. 96 (1988) and private communication.

9. V. S. Letokhov, in High Resolution Laser Spectroscopy, ed. K. Shimoda, Springer-Verlag, Berlin, p. 95 (1976). 
10. H. WaIther, in Laser Spectroscopy of Atoms and Molecules, ed. H. Walther, Springer-Verlag, Berlin, p. 99 (1976).

11. P. W. Smith and T. Hansch, Phys. Rev. Lett., 26, 740 (1971).

12. R. Vetter and P. R. Berman, Comments in Atomic and Molecular Physics, 10, 69 (1981).

13. C. Crépin, J.-L. Picqué, G. Rahmat, J. Vergés, R. Vetter, F.-X. Gadéa, M. Pélissier, F. Spiegelmann and J.-P. Malrieu, Chem. Phys. Lett., 110, 395 (1984).

14. G. Rahmat, J. Vergés, R. Vetter, F.-X. Gadéa, M. Pélissier and F. Spiegelmann, in Recent Advances in MolecuIar Reaction Dynamics, ed. R. Vetter and J. Vigué, Editions du C.N.R.S., Paris, p. 225 (1986).

15. G. Rahmat, F. SpiegeImann, J. Vergés and R. Vetter, Chem. Phys. Lett., 135, 459 (1987).

16. G. Rahmat, J. Vergés and R. Vetter, J. de Physique, Supp. no 12, 48, 601 (1987).

17. The Rabi frequency $f_{1}=4.8 \mathrm{MHz}$ is calculated with a laser power of $50 \mathrm{~mW}$, a beam diameter of 1.2 $\mathrm{mm}$, a dipole moment of 3.5 Debye (H. TeIIe, J. Chem. Phys., 81, 195, 1984) calculated at the internuclear distance $(5.4 \mathrm{a} . \mathrm{u}$.) of the molecular transition and a Franck-Condon factor of 0.17 .

18. R. Düren and E. Hasselbrink, J. Chem. Phys., 91, 5455 (1987).

19. F.-X. Gadéa, J.-M. L'Hermite, G. Rahmat and R. Vetter, Chem. Phys. Lett., 151, 183 (1988). 